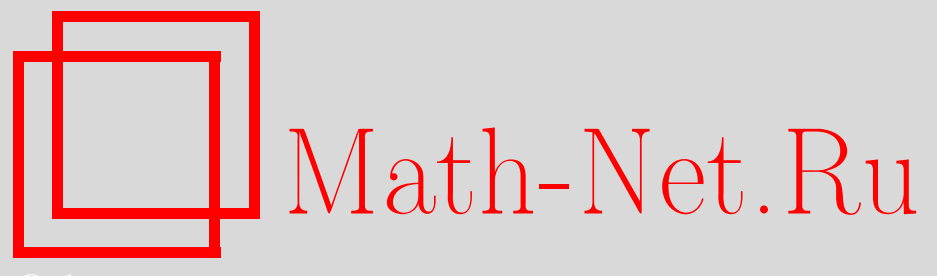

А. Н. Тимашёв, Случайные свободные деревья и леса с ограничениями на кратности вершин, Дискрет. матем., 2004, том 16, выпуск 4, 117-133

DOI: https://doi.org/10.4213/dm180

Использование Общероссийского математического портала Math-Net.Ru подразумевает, что вы прочитали и согласны с пользовательским соглашением http://www . mathnet.ru/rus/agreement

Параметры загрузки:

IP : 52.90 .164 .192

26 апреля 2023 г., $14: 55: 53$ 
УдК 519.2

\title{
Случайные свободные деревья \\ и леса с ограничениями \\ на кратности вершин
}

(c) 2004 г. А. Н. Тимашёв

\begin{abstract}
Рассматриваются свободные (некорневые) деревья с $n$ занумерованными вершинами, кратности которых принимают значения из некоторого фиксированного подмножества $A$ множества целых неотрицательных чисел такого, что $A$ содержит нуль, $A \neq\{0\}, A \neq\{0,1\}$ и наибольший общий делитель чисел $\{k \mid k \in A\}$ равен единице. Получена асимптотика числа всех таких деревьев при $n \rightarrow \infty$. В предположении, что на множестве этих деревьев задано равномерное распределение, для случайной величины $\mu_{r}^{(A)}(r \in A)$, равной числу вершин кратности $r$ в случайно выбранном дереве, найдены асимптотики математического ожидания и дисперсии при $n \rightarrow \infty$, а также доказаны локальная нормальная и пуассоновская теоремы для распределения вероятностей этой случайной величины. Для случая $A=\{0,1\}$ получены оценки чисел всех лесов с $n$ занумерованными вершинами, состоящих из $N$ свободных деревьев, при $n \rightarrow \infty$ и различных предположениях о функции $N=N(n)$. Найдена асимптотика числа всех лесов из свободных деревьев с $n$ вершинами, кратности которых не превосходят 1. Доказаны локальные нормальные и пуассоновские теоремы для числа деревьев заданного объема и общего числа деревьев в случайном лесе такого типа. Получены предельные теоремы, оценивающие распределение вероятностей случайной величины, равной объему дерева, содержащего вершину с фиксированным номером.
\end{abstract}

Рассмотрим свободное (некорневое) дерево с $n$ занумерованными вершинами. Число $d_{n}$ всех таких деревьев определяется формулой Кэли [1]

$$
d_{n}=n^{n-2} \text {. }
$$

Как обычно, степенью вершины дерева будем называть число инцидентных ей ребер. Кратность вершины на единицу меньше ее степени. Если $k_{1}, \ldots, k_{n}$ - кратности вершин свободного дерева с $n$ вершинами, $n \geqslant 2$, то

$$
k_{1}+\ldots+k_{n}=n-2 \text {. }
$$

Согласно алгоритму Прюфера [1] количество свободных деревьев с $n$ вершинами, занумерованными числами $1,2, \ldots, n$, с кратностями $k_{1}, \ldots, k_{n}$ соответственно, при условии (2) равно

$$
\frac{(n-2) !}{k_{1} ! \ldots k_{n} !}
$$


В формуле (3) $k_{1}, \ldots, k_{n} \in \mathbf{N}_{0}$, где $\mathbf{N}_{0}-$ множество всех целых неотрицательных чисел. Пусть $A$ - непустое подмножество $\mathbf{N}_{0}$. Всюду далее будем предполагать, что $A$ фиксировано, то есть выбор множества $A$ не зависит от $n$.

Пусть $d_{n}(A)$ - число всех свободных деревьев на $n$ вершинах, имеющих кратности, принадлежащие $A$. Тогда согласно (3)

$$
d_{n}(A)=(n-2) ! \sum_{k_{1}+\ldots+k_{n}=n-2, \quad k_{1}, \ldots, k_{n} \in A} \frac{1}{k_{1} ! \ldots k_{n} !} .
$$

Если, в частности, $A=\mathbf{N}_{0}$, то из (4) следует (1), то есть

$$
d_{n}\left(\mathbf{N}_{0}\right)=d_{n}=n^{n-2} .
$$

Заметим, что если $0 \notin A$, то $d_{n}(A)=0$, поскольку в любом дереве с конечным числом вершин $k_{1} \ldots k_{n}=0$.

Допустим, что множество $A$ удовлетворяет следующим условиям:

$$
\begin{gathered}
0 \in A, \quad A \neq\{0\}, \quad A \neq\{0,1\}, \\
\operatorname{gcd}\{k \mid k \in A\}=1,
\end{gathered}
$$

где $\operatorname{gcd}\{\cdot\}$ - наибольший общий делитель чисел, указанных в скобках.

Из (4) нетрудно получить, что (см. [2])

$$
\frac{d_{n}(A)}{(n-2) !}=\operatorname{coeff}_{z^{n-2}}\left\{\left(P_{A}(z)\right)^{n}\right\}
$$

где

$$
P_{A}(z)=\sum_{k \in A} \frac{z^{k}}{k !}
$$

Из (7) согласно интегральной формуле Коши следует, что

$$
d_{n}(A)=\frac{(n-2) !}{2 \pi i} \oint \frac{\left(P_{A}(z)\right)^{n}}{z^{n-1}} d z
$$

где интегрирование осуществляется по окружности с центром в нуле, пробегаемой в положительном направлении.

Далее будем считать, что все $d_{n}(A)$ деревьев рассматриваемого типа имеют одинаковые вероятности, равные $\left(d_{n}(A)\right)^{-1}$. Пусть $\mu_{r}^{(A)}, r \in A$, - число вершин кратности $r$ в случайно выбранном дереве. Если $A=\mathbf{N}_{0}$, то случайная вершина $\mu_{r}^{(A)}$ совпадает с числом ячеек, содержащих ровно $r$ частиц каждая, при случайном равновероятном размещении $n-2$ различных частиц по $n$ различным ячейкам, и ее предельные распределения хорошо изучены (см. [3]). В общем случае, с использованием (9) и метода перевала, в работе получена оценка чисел $d_{n}(A)$, найдены асимптотики математического ожидания и дисперсии случайной величины $\mu_{r}^{(A)}$ при $n \rightarrow \infty$ и условиях (5), (6), а также доказаны локальная нормальная и пуассоновская теоремы для распределения вероятностей этой случайной величины. Если $A=\{0,1\}$, то согласно (7)-(9)

$$
P_{A}(z)=1+z
$$


и при $n \geqslant 2$

$$
d_{n}(A)=\frac{n !}{2}
$$

При $n=1$

$$
d_{1}(A)=1
$$

Согласно (10) и (11) при $|z|<1$

$$
\sum_{n=1}^{\infty} \frac{d_{n}(A)}{n !} z^{n}=\frac{1}{2} z\left(1+(1-z)^{-1}\right)
$$

и если $L_{N}(n)$ - число всех лесов с $n$ занумерованными вершинами, состоящих из $N$ свободных деревьев с кратностями вершин, не превосходящими 1 (деревья без веток), то согласно [1]

$$
\sum_{n=N}^{\infty} L_{N}(n) \frac{z^{n}}{n !}=\frac{z^{N}}{2^{N} N !}\left(1+(1-z)^{-1}\right)^{N}, \quad N=1,2, \ldots
$$

и поэтому

$$
\begin{aligned}
L_{N}(n) & =\frac{n !}{2^{N} N !} \frac{1}{2 \pi i} \oint \frac{\left(1+(1-z)^{-1}\right)^{N}}{z^{n-N+1}} d z \\
& =\frac{n !}{2^{N} N !} \operatorname{coeff}_{z^{n-N}}\left\{\left(1+(1-z)^{-1}\right)^{N}\right\}, \quad N=1, \ldots, n .
\end{aligned}
$$

Интегрирование в (12) осуществляется по окружности с центром в нуле радиуса, меньшего 1 , пробегаемой в положительном направлении.

Пусть $L(n)$ - число всех лесов с $n$ занумерованными вершинами, состоящих из свободных деревьев с кратностями вершин, не превосходящими 1. Тогда из (12) следует, что

$$
L(n)=\sum_{N=1}^{n} L_{N}(n)=\frac{n !}{2 \pi i} \oint \frac{\exp \left((z / 2)\left(1+(1-z)^{-1}\right)\right)}{z^{n+1}} d z .
$$

Интегрирование в (13) производится по тому же пути, что и в (12).

В работе получены асимптотические оценки чисел $L(n)$ и $L_{N}(n)$ при $n \rightarrow \infty$ и $N=N(n)$, где функция $N(n)$ удовлетворяет одному из следующих условий:

$$
N \in \mathbf{N} \text { фиксировано; }
$$

$N \rightarrow \infty$ так, что

$$
n-N=o(\sqrt{n}) \geqslant 0
$$

$N \rightarrow \infty$ так, что

$$
1<\beta_{0} \leqslant \beta=n / N \leqslant \beta_{1}<\infty,
$$


где $\beta_{0}, \beta_{1}-$ постоянные;

$N \rightarrow \infty$ так, что

$$
0<\alpha_{0} \leqslant \alpha=N / \sqrt{n} \leqslant \alpha_{1}<\infty
$$

где $\alpha_{0}, \alpha_{1}$ - постоянные.

В предположении, что все $L(n)$ лесов рассматриваемого типа имеют одинаковые вероятности, равные $(L(n))^{-1}$, на основе полученных оценок доказаны предельные теоремы пуассоновского типа для случайной величины $\eta_{r}(n)$, равной числу деревьев объема $r$ в случайно выбранном лесе, а также локальная нормальная теорема, оцениваюшая распределение вероятностей случайной величины $v_{n}$, равной общему числу деревьев в таком лесе.

Далее рассматривается случайная величина $\eta_{r}(n, N)$, равная числу свободных деревьев объема $r$ в случайном лесе указанного типа с $n$ отмеченными вершинами, содержащем $N$ деревьев (все такие леса считаются имеющими одинаковые вероятности, равные $\left.\left(L_{N}(n)\right)^{-1}\right)$. Приводятся точные формулы для факториальных моментов этой случайной величины, с помощью которых найдены достаточные условия сходимости распределения $\eta_{r}(n, N)$ к пуассоновскому закону, справедливые в трех различных областях изменения параметров $n, N \rightarrow \infty$, а также к нормальному закону (в локальном варианте). Для случая, когда $A=\mathbf{N}_{0}$, асимптотические оценки величин $L_{N}(n)$ и условия такого типа ранее были получены в $[4,5]$. Заметим, что использовавшиеся в $[4,5]$ методы, как правило, отличны от применяемых нами методов при доказательствах соответствующих утверждений (в идейном плане общим приемом является использование обобщенной схемы размещения [6]).

Пусть $\xi_{n}$ - число вершин свободного дерева, содержащего вершину с фиксированным номером, в определенном выше случайном лесе с $n$ занумерованными вершинами, и пусть величина $\xi(n, N)$ имеет тот же смысл для аналогичного случайного леса с $n$ занумерованными вершинами и $N$ свободными деревьями. В заключительной части работы доказаны предельные теоремы, оценивающие распределения вероятностей случайных величин $\xi_{n}$ и $\xi(n, N)$.

Теорема 1. Пусть $n \rightarrow \infty$ и выполняются условия (5), (6). Тогда

$$
d_{n}(A)=\frac{n^{n-2} e^{-n}}{\sqrt{P_{A}^{\prime \prime}\left(z_{1}\right)}}\left(P_{A}\left(z_{1}\right)\right)^{n+1 / 2} z_{1}^{-(n-1)}\left(1+O\left(n^{-1}\right)\right),
$$

где функиия $P_{A}(z)$ определена в (8) $и z=z_{1}$ - единственный положительный корень уравнения

$$
z P_{A}^{\prime}(z)\left(P_{A}(z)\right)^{-1}=1
$$

Доказательство. Справедливость оценки (18) устанавливается с помощью метода перевала в варианте, изложенном на стр. 194 в [7], если учесть, что согласно (5) свободный член в разложении (8) равен 1. При условиях теоремы $1 \max _{|z|=z_{1}}\left|P_{A}(z)\right|$ достигается только при $z=z_{1}$.

Нетрудно проверить также, что $z_{1}$ - точка перевала первого порядка функции

$$
\omega_{1}(z)=\ln P_{A}(z)-\ln z
$$


(для логарифма выбираем ту непрерывную ветвь, которая принимает вещественные значения на вещественной положительной полуоси), поскольку согласно (19)

$$
\omega_{1}^{\prime}\left(z_{1}\right)=0, \quad \omega_{1}^{\prime \prime}\left(z_{1}\right)=P_{A}^{\prime \prime}\left(z_{1}\right)\left(P_{A}\left(z_{1}\right)\right)^{-1}>0 .
$$

Существование и единственность положительного корня уравнения (19) следуют из условий (5) и того факта, что функция

$$
f(z)=z P^{\prime}(z)\left(P_{A}(z)\right)^{-1}
$$

непрерывна и строго возрастает от 0 до $\sup A>1$ при возрастании $z$ от 0 до бесконечности.

Таким образом, в силу (6) интеграл в (9) асимптотически, с точностью до множителя $1+O\left(n^{-1}\right)$, равен вкладу точки перевала $z=z_{1}$. Вычисляя этот вклад стандартными методами, учитывая (9) и используя формулу Стирлинга, получаем (18).

Теорема 1 доказана.

Замечание 1. При условиях теоремы 1 остаточный член $O\left(n^{-1}\right)$ в (18) можно заменить на асимптотический степенной ряд вида $\sum_{m=1}^{\infty} A_{m} n^{-m}$, в котором коэффициенты $A_{m}$ не зависят от $n$. Вычисление этих коэффициентов, как правило, приводит к громоздким выкладкам. Для простоты изложения ограничимся случаем $m=1$. В этом случае можно показать, что

$$
A_{1}=\frac{13}{12}+\frac{P_{A}\left(z_{1}\right) P_{A}^{\prime \prime \prime}\left(z_{1}\right)}{z_{1}\left(P_{A}^{\prime \prime}\left(z_{1}\right)\right)^{2}}-\frac{2}{g_{A}\left(z_{1}\right)}+\frac{\alpha_{4}}{8\left(g_{A}\left(z_{1}\right)\right)^{2}}-\frac{5 \alpha_{3}^{2}}{24\left(g_{A}\left(z_{1}\right)\right)^{3}},
$$

где

$$
\begin{aligned}
g_{A}\left(z_{1}\right) & =z_{1}^{2} P_{A}^{\prime \prime}\left(z_{1}\right)\left(P_{A}\left(z_{1}\right)\right)^{-1}, \\
\alpha_{3} & =z_{1}^{3} P_{A}^{\prime \prime \prime}\left(z_{1}\right)\left(P_{A}\left(z_{1}\right)\right)^{-1}, \\
\alpha_{4} & =z_{1}^{4} P_{A}^{(4)}\left(z_{1}\right)\left(P_{A}\left(z_{1}\right)\right)^{-1}+2 \alpha_{3}+g_{A}\left(z_{1}\right)-3\left(g_{A}\left(z_{1}\right)\right)^{2} .
\end{aligned}
$$

Таким образом, остаточный член вида $O\left(n^{-1}\right)$ в (18) можно заменить на сумму $A_{1} n^{-1}+O\left(n^{-2}\right)$, и оценка (18) останется справедливой, если значение $A_{1}$ определяется согласно (20)-(23). Заметим, что в формулах (20)-(23)

$$
P_{A}^{(j)}\left(z_{1}\right)=\sum_{k \in A: k \geqslant j} \frac{z_{1}^{k-j}}{(k-j) !}, \quad j=1,2, \ldots
$$

Теорема 2. При $n \rightarrow \infty$ в условиях теоремы 1

$$
\begin{aligned}
& \mathbf{E} \mu_{r}^{(A)}=n p_{r}^{(A)}\left(z_{1}\right)\left(1+O\left(n^{-1}\right)\right), \\
& \mathbf{D} \mu_{r}^{(A)}=n \sigma_{r}^{2}\left(1+O\left(n^{-1}\right)\right),
\end{aligned}
$$

где $r \in A$,

$$
\sigma_{r}^{2}=p_{r}^{(A)}\left(z_{1}\right)\left(1-p_{r}^{(A)}\left(z_{1}\right)\left(1+\frac{(r-1)^{2}}{g_{A}\left(z_{1}\right)}\right)\right)>0,
$$

значение $g_{A}\left(z_{1}\right)$ определено в (21) $u$

$$
p_{r}^{(A)}\left(z_{1}\right)=\frac{z_{1}^{r}}{r ! P_{A}\left(z_{1}\right)}
$$


Доказательство. Заметим, что если $r \in A$, то

$$
\mathbf{E} \mu_{r}^{(A)}=n\left(\begin{array}{c}
n-2 \\
r
\end{array}\right) \frac{M_{n-2-r}(n-1 ; A)}{M_{n-2}(n ; A)}
$$

где

$$
M_{n}(N ; A)=\frac{n !}{2 \pi i} \oint \frac{\left(P_{A}(z)\right)^{N}}{z^{n+1}} d z
$$

В частности, согласно (9)

$$
M_{n-2}(n ; A)=d_{n}(A) \text {. }
$$

Применяя метод перевала и рассуждая так же, как при доказательстве теоремы 1 , из (18), (29), (30), получаем, что при $n \rightarrow \infty$

$$
M_{n-k}(n-l ; A)=\frac{(n-k) ! d_{n}(A) z_{1}^{k-2}}{(n-2) !\left(P_{A}\left(z_{1}\right)\right)^{l}}\left(1+O\left(n^{-1}\right)\right), \quad k, l=0,1, \ldots
$$

Полагая в (31) $k=r+2, l=1$, подставляя получившееся значение $M_{n-r-2}(n-1 ; A)$ в (28) и учитывая (27), (30), выводим (24).

Далее, аналогично (28),

$$
\mathbf{E} \mu_{r}^{(A)}\left(\mu_{r}^{(A)}-1\right)=n(n-1)\left(\begin{array}{c}
n-2 \\
r
\end{array}\right)\left(\begin{array}{c}
n-2-r \\
r
\end{array}\right) \frac{M_{n-2-2 r}(n-2 ; A)}{M_{n-2}(n ; A)} .
$$

Согласно (27), (31) отсюда следует, что

$$
\mathbf{E} \mu_{r}^{(A)}\left(\mu_{r}^{(A)}-1\right)=n^{2}\left(p_{r}^{(A)}\left(z_{1}\right)\right)^{2}\left(1+O\left(n^{-1}\right)\right) .
$$

Однако для нахождения асимптотики дисперсии $\mathbf{D} \mu_{r}^{(A)}$ этих равенств недостаточно, поскольку из них с учетом (24) следует только, что

$$
\mathbf{D} \mu_{r}^{(A)}=O(n)
$$

Чтобы получить (25), (26), нужно найти асимптотику чисел $M_{n-2-r}(n-1 ; A)$, $M_{n-2-2 r}(n-2 ; A)$ с остаточными членами порядка $O\left(n^{-2}\right)$. Соответствующие (достаточно громоздкие) выкладки мы опускаем.

Покажем, что $\sigma_{r}^{2}>0$. Рассмотрим случайную величину $\xi_{1}$, для которой

$$
\mathbf{P}\left\{\xi_{1}=k\right\}=p_{k}^{(A)}\left(z_{1}\right)>0, \quad k \in A
$$

Легко подсчитать, что

$$
\mathbf{E} \xi_{1}=\frac{z_{1} P_{A}^{\prime}\left(z_{1}\right)}{P_{A}\left(z_{1}\right)}=1
$$

в силу (19) и

$$
\mathbf{D} \xi_{1}=g_{A}\left(z_{1}\right)>0
$$

согласно (5), (21). 
Далее, рассмотрим случайную величину $\xi_{1}^{(r)}, r \in A$, для которой

$$
\begin{aligned}
\mathbf{P}\left\{\xi_{1}^{(r)}=k\right\} & =\mathbf{P}\left\{\xi_{1}=k \mid \xi_{1} \neq r\right\} \\
& =\frac{z_{1}^{k}}{k !\left(P_{A}\left(z_{1}\right)-z_{1}^{r} / r !\right)}>0, \quad k \in A, \quad k \neq r .
\end{aligned}
$$

Непосредственно проверяется, что с учетом (26)

$$
\mathbf{E} \xi_{1}^{(r)}=\frac{1-r p_{r}^{(A)}\left(z_{1}\right)}{1-p_{r}^{(A)}\left(z_{1}\right)} .
$$

При условиях (5), (6) множество $A$ не может быть двухэлементным, поэтому случайная величина $\xi_{1}^{(r)}$ имеет невырожденное распределение, то есть $\mathbf{D} \xi_{1}^{(r)}>0$. С другой стороны, легко показать, что

$$
\mathbf{D} \xi_{1}^{(r)}=\frac{g_{A}\left(z_{1}\right) \sigma_{r}^{2}}{p_{r}^{(A)}\left(z_{1}\right)\left(1-p_{r}^{(A)}\left(z_{1}\right)\right)^{2}} .
$$

Следовательно, если $r \in A$ то $\sigma_{r}^{2}>0$.

Теорема доказана.

Теорема 3. При $n \rightarrow \infty$ в условиях теоремы 1 равномерно относительно цельх положительных значений $m=n p_{r}^{(A)}\left(z_{1}\right)+y \sqrt{n}$, для которых величина у заключена в любом фиксированном конечном интервале,

$$
\mathbf{P}\left\{\mu_{r}^{(A)}=m\right\}=\frac{d_{r}}{\sqrt{2 \pi n \sigma_{r}^{2}}} \exp \left(-\frac{y^{2}}{2 \sigma_{r}^{2}}\right)(1+O(1 / \sqrt{n})),
$$

где $r \in A$, значение $\sigma_{r}^{2}$ определено в (26),

$$
\begin{aligned}
d_{0} & =\operatorname{gcd}\left\{k-\min A_{0} \mid k \in A_{0}\right\}, \\
d_{r} & =\operatorname{gcd}\left\{k \mid k \in A_{r}\right\}, \quad r=1,2, \ldots, \\
A_{r} & =A \backslash\{r\}, \quad r=0,1, \ldots,
\end{aligned}
$$

$\operatorname{gcd}\{\cdot\}$ - наибольший общий делитель множества натуральных чисел, стоящего в фигурнblx скобках.

Доказательство. Для обоснования оценки (34) используем метод, позволяющий свести изучение асимптотического поведения вероятности $\mathbf{P}\left\{\mu_{r}^{(A)}=m\right\}$ к применению классической локальной нормальной предельной теоремы для последовательностей независимых одинаково распределенных целочисленных случайных величин [3]. Рассмотрим последовательность независимых одинаково распределенных случайных величин $\xi_{1}, \xi_{2}, \ldots, \xi_{n}, \ldots$, предполагая, что случайная величина $\xi_{1}$ имеет указанное в доказательстве теоремы 2 распределение. По локальной нормальной теореме

$$
\begin{aligned}
\mathbf{P}\left\{\xi_{1}+\ldots+\xi_{n}=n-2\right\} & =\frac{1}{\sqrt{2 \pi n \mathbf{D} \xi_{1}}}(1+O(1 / \sqrt{n})) \\
& =\frac{1}{\sqrt{2 \pi n g_{A}\left(z_{1}\right)}}(1+O(1 / \sqrt{n})) .
\end{aligned}
$$


Далее рассмотрим последовательность независимых одинаково распределенных случайных величин $\xi_{1}^{(r)}, \ldots, \xi_{n-m}^{(r)}, \ldots$ таких, что случайная величина $\xi_{1}^{(r)}$ имеет указанное выше распределение. При наших условиях из (32) следует, что

$$
\frac{n-2-m r}{n-m}=\mathbf{E} \xi_{1}^{(r)}(1+O(1 / \sqrt{n}))
$$

поскольку величина $\mathbf{E} \xi_{1}^{(r)}$ ограничена.

Применяя локальную нормальную теорему к последовательности $\xi_{1}^{(r)}, \ldots, \xi_{n-m}^{(r)}$, можно утверждать, что

$$
\begin{aligned}
& \mathbf{P}\left\{\xi_{1}^{(r)}+\ldots+\xi_{n-m}^{(r)}=n-2-\right.m r\}=\frac{d_{r}}{\sqrt{2 \pi n\left(1-p_{r}^{(A)}\left(z_{1}\right)\right) \mathbf{D} \xi_{1}^{(r)}}} \\
& \times \exp \left(-\frac{(r-1)^{2} y^{2}}{2\left(1-p_{r}^{(A)}\left(z_{1}\right)\right)^{3} \mathbf{D} \xi_{1}^{(r)}}\right)(1+O(1 / \sqrt{n})),
\end{aligned}
$$

поскольку случайные величины $\xi_{1}^{(r)}, \ldots, \xi_{n-m}^{(r)}$ принимают значения на целочисленной решетке с максимальным шагом $d_{r}$.

Согласно локальной теореме Муавра-Лапласа

$$
\begin{aligned}
\left(\begin{array}{c}
n \\
m
\end{array}\right)\left(p_{r}^{(A)}\left(z_{1}\right)\right)^{m}\left(1-p_{r}^{(A)}\left(z_{1}\right)\right)^{n-m} & =\frac{1}{\sqrt{2 \pi n p_{r}^{(A)}\left(z_{1}\right)\left(1-p_{r}^{(A)}\left(z_{1}\right)\right)}} \\
& \times \exp \left(-\frac{y^{2}}{2 p_{r}^{(A)}\left(z_{1}\right)\left(1-p_{r}^{(A)}\left(z_{1}\right)\right)}\right)(1+O(1 / \sqrt{n})) .
\end{aligned}
$$

Поскольку $\mathbf{P}\left\{\xi_{1}=r\right\}=p_{r}^{(A)}\left(z_{1}\right)$ при $r \in A$, аналогично тому, как это сделано в [3], получаем, что

$$
\mathbf{P}\left\{\mu_{r}^{(A)}=m\right\}=\left(\begin{array}{l}
n \\
m
\end{array}\right)\left(p_{r}^{(A)}\left(z_{1}\right)\right)^{m}\left(1-p_{r}^{(A)}\left(z_{1}\right)\right)^{n-m} \frac{\mathbf{P}\left\{\xi_{1}^{(r)}+\ldots+\xi_{n-m}^{(r)}=n-2-m r\right\}}{\mathbf{P}\left\{\xi_{1}+\ldots+\xi_{n}=n-2\right\}} .
$$

Из формул (26), (33), (35)-(38) следует (34). Теорема доказана.

Следствие 1. При $n \rightarrow \infty$ в условиях теоремы 3 случайная величина

$$
\frac{\mu_{r}^{(A)}-n p_{r}^{(A)}\left(z_{1}\right)}{\sqrt{n \sigma_{r}^{2}}}
$$

распределена в пределе по нормальному закону.

Теорема 4. Пусть при условиях теоремы $1 A$ - счетное множество $u r \rightarrow \infty u$ $n=n(r) \rightarrow \infty$ так, что $r \in A$ и $n p_{r}^{(A)}\left(z_{1}\right) \rightarrow \lambda>0$. Тогда случайная величина $\mu_{r}^{(A)}$ распределена в пределе по закону Пуассона с параметром $\lambda$. 
Доказательство. Используя (24), получаем, что $\mathbf{E} \mu_{r}^{(A)} \rightarrow \lambda$. Аналогично (28) можно утверждать, что при $j=1,2, \ldots$

$$
\mathbf{E}\left(\mu_{r}^{(A)}\right)_{j}=\frac{n !(n-2) !}{(n-j) !(n-2-r j) !(r !)^{j}} \frac{M_{n-2-r_{j}}(n-j ; A)}{d_{n}(A)},
$$

где значение $M_{n-2-r j}(n-j ; A)$ определяется в (29) с заменой $n$ на $n-2-r j$ и $N$ на $n-j$. Поскольку при наших условиях $r \ln r \sim \ln n$, то $r \sim \ln n / \ln \ln n$, и поэтому при $k=2+r j$ и $l=j$ формула (31) остается справедливой с заменой остаточного члена вида $O\left(n^{-1}\right)$ на $o(1)$. Следовательно,

$$
M_{n-2-r j}(n-j ; A)=\frac{(n-2-r j) ! z_{1}^{r j} d_{n}(A)}{(n-2) !\left(P_{A}\left(z_{1}\right)\right)^{j}}(1+o(1)) .
$$

Из (27), (39), (40) получаем, что

$$
\mathbf{E}\left(\mu_{r}^{(A)}\right)_{j}=\left(n p_{r}^{(A)}\left(z_{1}\right)\right)^{j}(1+o(1))=\lambda^{j}(1+o(1)), \quad j=1,2, \ldots
$$

Теорема 4 доказана.

Всюду далее будем предполагать, что $A=\{0,1\}$. Если $|z|<1$, то

$$
\left(1+(1-z)^{-1}\right)^{N}=\left(2+\sum_{k=1}^{\infty} z^{k}\right)^{N}
$$

и при $N=1, \ldots, n-1$

$$
\operatorname{coeff}_{z^{n-N}}\left\{\left(1+(1-z)^{-1}\right)^{N}\right\}=\sum_{m=\max \{0,2 N-n\}}^{N-1}\left(\begin{array}{l}
N \\
m
\end{array}\right) 2^{m}\left(\begin{array}{l}
n-N-1 \\
N-m-1
\end{array}\right) .
$$

Предложение 1. Пусть $n \rightarrow \infty$ и выполнено условие (14), тогда

$$
L_{N}(n)=\frac{n ! n^{N-1}}{2^{N} N !(N-1) !}\left(1+O\left(n^{-1}\right)\right) .
$$

Доказательство. При всех достаточно больших значениях $n$ справедливо неравенство $N<n / 2$, и поэтому в сумме (41) будет $N$ слагаемых. Следовательно, при фиксированном $N \in \mathbf{N}$

$$
\begin{aligned}
\operatorname{coeff}_{z^{n-N}}\left\{\left(1+(1-z)^{-1}\right)^{N}\right\} & =\sum_{m=0}^{N-1}\left(\begin{array}{l}
N \\
m
\end{array}\right) 2^{m} \frac{n^{N-m-1}}{(N-m-1) !}\left(1+O\left(n^{-1}\right)\right) \\
& =\frac{n^{N-1}}{(N-1) !}\left(1+O\left(n^{-1}\right)\right),
\end{aligned}
$$

и оценка (42) следует из (12). Предложение 1 доказано.

Предложение 2. Пусть $n, N \rightarrow \infty$ и выполнено условие (16), тогда равномерно относительно $\beta \in\left[\beta_{0}, \beta_{1}\right]$

$$
L_{N}(n)=\frac{n !\left(\beta^{2}+4 \beta-4\right)^{-1 / 4}\left(1-z_{0} / 2\right)^{N}}{N ! \sqrt{2 \pi(n-N)} z_{0}^{n-N}\left(1-z_{0}\right)^{N}}\left(1+O\left(n^{-1}\right)\right),
$$


где

$$
z_{0}=\frac{3 \beta-2-\sqrt{\beta^{2}+4 \beta-4}}{2(\beta-1)} \in(0,1) .
$$

Доказательство. Нетрудно проверить, что $0<z_{0}<1$ при $\beta>1$. Используем (12), интегрируя по окружности $|z|=z_{0}$, пробегаемой в положительном направлении. Из (12) следует, что

$$
L_{N}(n)=\frac{n !}{2^{N} N !} \frac{1}{2 \pi i} \oint_{|z|=z_{0}} \varphi(z) \exp \left(N \omega_{0}(z)\right) d z,
$$

где

$$
\begin{aligned}
\varphi(z) & =z^{-1} \\
\omega_{0}(z) & =\ln \left(1+(1-z)^{-1}\right)-(\beta-1) \ln z .
\end{aligned}
$$

При этом согласно (44)

$$
\begin{aligned}
& \omega_{0}^{\prime}\left(z_{0}\right)=0, \\
& \omega_{0}^{\prime \prime}\left(z_{0}\right)=z_{0}^{-2}(\beta-1) \sqrt{\beta^{2}+4 \beta-4}>0 .
\end{aligned}
$$

Таким образом, $z_{0}$ - точка перевала первого порядка функции $\omega_{0}$. Кроме того, $\max _{\left.|z|=z_{0}\right)}\left|1+(1-z)^{-1}\right|$ достигается только при $z=z_{0}$. Дальнейшие рассуждения, имеющие целью обоснование оценки (43), аналогичны проведенным при доказательстве теоремы 1. Предложение 2 доказано.

Предложение 3. Если $n, N \rightarrow \infty$ так, что выполнено условие (15), то

$$
L_{N}(n)=\frac{n^{2(n-N)}}{2^{n-N}(n-N) !}(1+o(1)) .
$$

Доказательство. Допустим сначала, что величина $n-N$ ограничена. Тогда согласно (41) при $N \leqslant n-1$

$$
\begin{aligned}
\operatorname{coeff}_{z^{n-N}}\left\{\left(1+(1-z)^{-1}\right)^{N}\right\} & =\sum_{m=2 N-n}^{N-1}\left(\begin{array}{l}
N \\
m
\end{array}\right) 2^{m}\left(\begin{array}{c}
n-N-1 \\
N-m-1
\end{array}\right) \\
& =\frac{N^{n-N} 2^{2 N-n}}{(n-N) !}\left(1+O\left(n^{-1}\right)\right),
\end{aligned}
$$

и оценка (45) следует из (12).

Пусть $n-N \rightarrow \infty$, тогда из (15) выводим, что

$$
\beta=n / N=1+o(1 / \sqrt{N}), \quad(\beta-1) N \rightarrow \infty .
$$

Полагая $\varepsilon_{N}=\beta-1$, легко находим, что

$$
\varepsilon_{N}>0, \quad \varepsilon_{N}=o(1 / \sqrt{N}), \quad N \varepsilon_{N} \rightarrow \infty .
$$

Вычисляя значение $z_{0}$ согласно (44), после элементарных преобразований приходим к равенству

$$
z_{0}=2 \varepsilon_{N}-6 \varepsilon_{N}^{2}+O\left(\varepsilon_{N}^{3}\right)
$$


Из (46) с учетом соотношений

$$
N \varepsilon_{N}=n-N, \quad N \varepsilon_{N}^{2}=o(1)
$$

получаем, что

$$
\begin{aligned}
N\left(\ln \left(1-\left(z_{0} / 2\right)\right)-\ln \left(1-z_{0}\right)\right. & \left.-\varepsilon_{N} \ln z_{0}\right) \\
& =(n-N)(1-\ln 2-\ln (n-N)+\ln N)+o(1) .
\end{aligned}
$$

Подставляя эти оценки в (43), используя формулу Стирлинга для $(n-N)$ ! и замечая, что при условии (15)

$$
\frac{n !}{N !}=\left(\frac{n^{2}}{N}\right)^{n-N}(1+o(1))
$$

получаем (45). Предложение 3 доказано.

Предложение 4. Если $n, N \rightarrow \infty$ так, что выполнено условие (17), то равномерно относительно $\alpha \in\left[\alpha_{0}, \alpha_{1}\right]$

$$
L_{N}(n)=(n-1) ! 2^{-N} \alpha^{-2 N} \frac{1}{\sqrt{2 \pi}} \exp \left(2 N+\frac{\alpha^{2}}{2}-1\right)(1+o(1))
$$

Доказательство. При наших условиях

$$
\beta=\frac{n}{N}=\frac{\sqrt{n}}{\alpha} \rightarrow \infty
$$

и уравнение для определения точки перевала имеет вид

$$
\frac{z}{(1-z)(2-z)}=\beta-1=\frac{\sqrt{n}}{\alpha}-1 \text {. }
$$

Для каждого $n>\alpha^{2}$ оно имеет единственный корень $z=z_{n}^{*} \in(0,1)$. При $n \rightarrow \infty$ равномерно относительно $\alpha \in\left[\alpha_{0}, \alpha_{1}\right]$

$$
z_{n}^{*}=1-\frac{\alpha}{\sqrt{n}}+\frac{\alpha^{2}}{n}+O\left(1 / n^{3 / 2}\right) .
$$

Из этого соотношения и (17) следует, что

$$
\left(1+\left(1-z_{n}^{*}\right)^{-1}\right)^{N}\left(z_{n}^{*}\right)^{-(n-N+1)}=N^{N} \alpha^{-2 N} \exp \left(N+\frac{\alpha^{2}}{2}\right)(1+o(1))
$$

Кроме того,

$$
\omega_{0}^{\prime \prime}\left(z_{n}^{*}\right)=\frac{n}{\alpha^{2}}(1+o(1)),
$$

где функция $\omega_{0}$ определена при доказательстве предложения 2. С использованием полученных оценок формула (47) выводится аналогично (43). Предложение 4 доказано. 
Предложение 5. При $n \rightarrow \infty$

$$
L(n)=n !(2 e n)^{-3 / 4} \exp (\sqrt{2 n})(1+o(1)) .
$$

Доказательство. Чтобы получить (48), используем (13). Справедливо равенство

$$
L(n)=\frac{n !}{2 \pi i} \oint z^{-1} \exp (n \omega(z)) d z,
$$

где

и

$$
\omega(z)=\frac{z}{2 n}\left(1+(1-z)^{-1}\right)-\ln z
$$

если

$$
\omega^{\prime}(z)=\frac{1}{2 n}\left(1+(1-z)^{-2}\right)-z^{-1}=0,
$$

$$
z\left(1+(1-z)^{-2}\right)=2 n
$$

Уравнение (49) при любом $n=1,2, \ldots$ имеет единственный корень $z=z_{n} \in(0,1)$. Нетрудно показать, что при $n \rightarrow \infty$

$$
z_{n}=1-\frac{1}{\sqrt{2 n}}+\frac{1}{4 n}-\frac{1}{4 n \sqrt{2 n}}+o\left(n^{-3 / 2}\right) .
$$

Тогда

$$
\begin{aligned}
z_{n}^{-n} & =\exp (\sqrt{n / 2})(1+o(1)), \\
\frac{z_{n}}{2\left(1-z_{n}\right)} & =\sqrt{n / 2}-1 / 4+o(1), \\
\omega^{\prime \prime}\left(z_{n}\right) & =2 \sqrt{2 n}(1+o(1)) .
\end{aligned}
$$

Из этих оценок и (13) с помощью стандартных рассуждений выводим (48). Предложение 5 доказано.

Замечание 2. Если положить $l_{n}=L(n) / n !, n=0,1, \ldots$, то согласно (13) при $|z|<1$

$$
\sum_{n=0}^{\infty} l_{n} z^{n}=\exp \left((z / 2)\left(1+(1-z)^{-1}\right)\right) \text {. }
$$

Из этого соотношения нетрудно получить, что числа $l_{n}$ удовлетворяют линейным рекуррентным соотношениям

$$
l_{n}=\frac{l_{n-1}}{n}+\frac{1}{2} \sum_{k=0}^{n-2}(1-k / n) l_{k}, \quad n=2,3, \ldots,
$$

с начальными условиями $l_{0}=l_{1}=1$. При $n \rightarrow \infty$ согласно (48)

$$
l_{n}=(2 e n)^{-3 / 4} \exp (\sqrt{2 n})(1+o(1)) .
$$

Теорема 5. При $n \rightarrow \infty$ случайная величина $\eta_{r}(n), r=2,3, \ldots$, распределена в пределе по закону Пуассона с параметром 1/2. Случайная величина $\eta_{1}(n)$ распределена в пределе по закону Пуассона с параметром 1. 
Доказательство. Факториальные моменты случайной величины $\eta_{r}(n)$ имеют вид

$$
\mathbf{E}\left(\eta_{r}(n)\right)_{j}=\frac{n !\left(L_{1}(r)\right)^{j} L(n-r j)}{(n-r j) !(r !)^{j} L(n)}, \quad r, j=1,2, \ldots, \quad r j \leqslant n .
$$

Если $r \geqslant 2$, то согласно (10)

$$
L_{1}(r)=d_{r}(A)=\frac{r !}{2},
$$

где $A=\{0,1\}$. При $n \rightarrow \infty$ из (48) следует, что

$$
\frac{L(n-r j)}{L(n)}=\frac{(n-r j) !}{n !}(1+o(1)) .
$$

Подставляя эти оценки в (50), получаем, что при $r \geqslant 2$ и $n \rightarrow \infty$

$$
\mathbf{E}\left(\eta_{r}(n)\right)_{j}=2^{-j}(1+o(1)), \quad j=1,2, \ldots
$$

Если же $r=1$, то согласно (11) $L_{1}(1)=d_{1}(A)=1$, и поэтому при $n \rightarrow \infty$

$$
\mathbf{E}\left(\eta_{1}(n)\right)_{j}=1+o(1), \quad j=1,2, \ldots
$$

Теорема доказана.

Теорема 6. При $n \rightarrow \infty$ равномерно относительно челых положительных значений $N=\sqrt{n / 2}+x(n / 8)^{1 / 4}$, для которых величина х лежит в любом фиксированном конечном интервале,

$$
\mathbf{P}\left\{v_{n}=N\right\}=\frac{1}{\sqrt{\pi}}(2 / n)^{1 / 4} e^{-x^{2} / 2}(1+o(1)) .
$$

Доказательство. Полагая

$$
\alpha=\frac{N}{\sqrt{n}}=\frac{1}{\sqrt{2}}\left(1+\frac{x}{(2 n)^{1 / 4}}\right)
$$

и используя (47), нетрудно получить оценку

$$
L_{N}(n)=(n-1) ! \frac{1}{\sqrt{2 \pi}} \exp \left(\sqrt{2 n}-\frac{x^{2}}{2}-\frac{3}{4}\right)(1+o(1)) .
$$

Из формулы

$$
\mathbf{P}\left\{v_{n}=N\right\}=\frac{L_{N}(n)}{L(n)}
$$

и соотношений (48), (52) следует (51).

Теорема 6 доказана.

Замечание 3. Можно показать, что при $n \rightarrow \infty$

$$
\begin{aligned}
& \mathbf{E} v_{n}=\sqrt{\frac{n}{2}}(1+o(1)), \\
& \mathbf{D} v_{n}=\sqrt{\frac{n}{8}}(1+o(1)) .
\end{aligned}
$$

С учетом этих равенств и (51) получаем, что случайная величина $\left(v_{n}-\sqrt{n / 2}\right) /(n / 8)^{1 / 4}$ распределена в пределе при $n \rightarrow \infty$ по стандартному нормальному закону.

5 Дискретная математика, т.16 №4 
Теорема 7. Пусть $n, N \rightarrow \infty$ так, что $N^{2} / n \rightarrow \lambda>0$. Тогда спучайная величина $\eta_{r}(n, N), r=2,3, \ldots$, распределена в пределе по закону Пуассона с параметром $\lambda$. Случайная величина $\eta_{1}(n, N)$ распределена в пределе по закону Пуассона с параметром $2 \lambda$.

Доказательство. Факториальные моменты случайной величины $\eta_{r}(n, N)$ имеют вид [8]

$$
\mathbf{E}\left(\eta_{r}(n, N)\right)_{j}=\frac{n !\left(L_{1}(r)\right)^{j} L_{N-j}(n-r j)}{(n-r j) !(r !)^{j} L_{N}(n)}, \quad r, j=1,2, \ldots, \quad(r-1) j \leqslant n-N .
$$

Если $\alpha=N / \sqrt{n}$, то $\alpha^{2}=\lambda+o(1)$ при $n \rightarrow \infty$ и справедлива оценка (47). Используя (10), (11), (47), (53), при $r \geqslant 2$ получаем, что

$$
\mathbf{E}\left(\eta_{r}(n, N)\right)_{j}=\alpha^{2 j}(1+o(1))=\lambda^{j}(1+o(1)), \quad j=1,2, \ldots
$$

При $r=1$ аналогично доказывается, что

$$
\mathbf{E}\left(\eta_{1}(n, N)\right)_{j}=2^{j} \alpha^{2 j}(1+o(1))=(2 \lambda)^{j}(1+o(1)), \quad j=1,2, \ldots
$$

Теорема 7 доказана.

Теорема 8. Пусть $n, N, r \rightarrow \infty$ так, что выполнено условие (16) $u$

$$
(n-N) z_{0}^{r}\left(z_{0}^{-1}-1\right)^{2} \rightarrow \lambda>0,
$$

где $z_{0}$ определено в (44). Тогда случайная величина $\eta_{r}(n, N)$ распределена в пределе по закону Пуассона с параметром $\lambda$.

Доказательство. Из (54) следует, что

$$
r=-\frac{\ln N}{\ln z_{0}}(1+o(1))=O(\ln N),
$$

и поэтому оценка (43) при замене $N$ на $N-j$ и $n$ на $n-r j$ останется справедливой, если остаточный член вида $O\left(n^{-1}\right)$ заменить на $o(1)$. Подставляя оценки чисел $L_{N}(n)$ и $L_{N-j}(n-r j)$ в (53), приходим к равенствам

$$
\mathbf{E}\left(\eta_{r}(n, N)\right)_{j}=N^{j}\left(1+\left(1-z_{0}\right)^{-1}\right)^{-j} z_{0}^{(r-1) j}(1+o(1)), \quad j==1,2, \ldots
$$

В силу (44)

$$
\frac{z_{0}}{\left(1-z_{0}\right)\left(2-z_{0}\right)}=\beta-1=\frac{n-N}{N} \text {, }
$$

поэтому согласно (54)

$$
\mathbf{E}\left(\eta_{r}(n, N)\right)_{j}=(n-N)^{j} z_{0}^{r j}\left(z_{0}^{-1}-1\right)^{2 j}(1+o(1))=\lambda^{j}(1+o(1)), \quad j=1,2, \ldots
$$

Теорема 8 доказана.

Теорема 9. Пусть $n, N \rightarrow \infty$ так, что

$$
n(1-N / n)^{r-1} \rightarrow \frac{\lambda}{2^{r-2}}>0 .
$$

Тогда случайная величина $\eta_{r}(n, N), r=3,4, \ldots$, распределена в пределе по закону Пуассона с параметром $\lambda$. 
Доказательство. При $r \geqslant 3$ и условии (55) $n-N \rightarrow \infty$, и неравенство $(r-1) j \leqslant n-N$ будет выполняться при всех достаточно больших значениях $n$ и $N$, поэтому для таких $n$ и $N$ формула (53) справедлива. Полагая

$$
\beta=\frac{n}{N}=1+\delta_{N}
$$

выводим из (55), что

$$
\delta_{N}=\lambda^{1 /(r-1)} 2^{-(r-2) /(r-1)} N^{-1 /(r-1)}(1+o(1)) .
$$

Заменяя в (46) $\varepsilon_{N}$ на $\delta_{N}=o(1)$, получаем, что

$$
z_{0}=2 \delta_{N}\left(1+O\left(\delta_{N}\right)\right)=(2 \lambda)^{1 /(r-1)} N^{-1 /(r-1)}\left(1+O\left(N^{-1 /(r-1)}\right)\right) .
$$

Рассуждая так же, как и при доказательстве теоремы 8, находим, что

$$
\mathbf{E}\left(\eta_{r}(n, N)\right)_{j}=N^{j}\left(1+\left(1-z_{0}\right)^{-1}\right)^{-j} z_{0}^{(r-1) j}(1+o(1))=\lambda^{j}(1+o(1)), \quad j=1,2, \ldots
$$

Теорема 9 доказана.

Замечание 4. При $r=2$ и при выполнении условий теоремы 9 случайная величина $\eta_{2}(n, N)$ асимптотически вырождена в точке $\lambda \in \mathbf{N}$.

Теорема 10. Пусть $n, N \rightarrow \infty$ так, что выполнено условие (16). Тогда равномерно относительно $\beta \in\left[\beta_{0}, \beta_{1}\right]$ и иелых положительных значений $m=N p_{r}\left(z_{0}\right)+y \sqrt{N}$, для которых величина у лежит в любом фиксированном конечном интервале,

$$
\mathbf{P}\left\{\eta_{r}(n, N)=m\right\}=\frac{1}{\sqrt{2 \pi N \sigma^{2}}} \exp \left(-\left(y^{2} / 2 \sigma^{2}\right)(1+O(1 / \sqrt{N}))\right),
$$

где

$$
\begin{aligned}
p_{r}\left(z_{0}\right) & =\frac{z_{0}^{r-1}\left(1-z_{0}\right)}{2-z_{0}}, \quad r=2,3, \ldots, \\
p_{1}\left(z_{0}\right) & =\frac{2\left(1-z_{0}\right)}{2-z_{0}}, \\
\sigma^{2} & =p_{r}\left(z_{0}\right)\left(1-p_{r}\left(z_{0}\right)\left(1+\frac{(\beta-r)^{2} z_{0}}{(\beta-1)^{2}\left(2-z_{0}^{2}\right)}\right)\right)>0,
\end{aligned}
$$

величина $z_{0}$ определена в (44).

Теорема 10 доказывается аналогично теореме 3 (это доказательство в идейном плане не содержит ничего нового, и мы его опускаем).

Замечание 5. Можно показать, что при условиях теоремы 10 равномерно относительно $\beta \in\left[\beta_{0}, \beta_{1}\right]$

$$
\begin{aligned}
& \mathbf{E} \eta_{r}(n, N)=N p_{r}\left(z_{0}(1+O(1 / \sqrt{N})),\right. \\
& \mathbf{D} \eta_{r}(n, N)=N \sigma^{2}(1+O(1 / \sqrt{N})) .
\end{aligned}
$$

Из этих равенств и (56) следует, что случайная величина

$$
\frac{\eta_{r}(n, N)-N p_{r}\left(z_{0}\right)}{\sqrt{N \sigma^{2}}}
$$

распределена в пределе по стандартному нормальному закону. 
Теорема 11. При условиях теоремы 10

$$
\begin{aligned}
& \mathbf{P}\{\xi(n, N)=k\}=\frac{k z_{0}^{k-1}\left(1-z_{0}\right)}{\beta\left(2-z_{0}\right)}-\left(1+O\left(n^{-1}\right)\right), \quad k=2,3, \ldots, \\
& \mathbf{P}\{\xi(n, N)=1\}=\frac{2\left(1-z_{0}\right)}{\beta\left(2-z_{0}\right)}\left(1+O\left(n^{-1}\right)\right) .
\end{aligned}
$$

Доказательство. Согласно определению случайной величины $\xi(n, N)$ справедливы равенства

$$
\mathbf{P}\{\xi(n, N)=k\}=\frac{\left(\begin{array}{c}
n-1 \\
k-1
\end{array}\right) L_{1}(k) L_{N-1}(n-k)}{L_{N}(n)}, \quad k=1, \ldots, n .
$$

Из (12), (43), (44) следует, что

$$
\frac{L_{N-1}(n-k)}{L_{N}(n)}=\frac{2\left(1-z_{0}\right) z_{0}^{k-1}}{\beta n^{k-1}\left(2-z_{0}\right)}(1+O(1 / n)), \quad k=1,2, \ldots
$$

Подставляя эти оценки в (59) и учитывая (10), (11), получаем (57), (58).

Теорема 11 доказана.

Теорема 12. При любом фиксированном $x>0$

$$
\lim _{n \rightarrow \infty} \mathbf{P}\left\{\xi_{n}<x \sqrt{2 n}\right\}=1-(x+1) e^{-x} .
$$

Доказательство. Аналогично (59)

$$
\mathbf{P}\left\{\xi_{n}=k\right\}=\frac{\left(\begin{array}{c}
n-1 \\
k-1
\end{array}\right) L_{1}(k) L(n-k)}{L(n)}, \quad k=1, \ldots, n .
$$

Утверждение теоремьі означает, что при $n \rightarrow \infty$ последовательность распределений случайных величин $\xi_{n} / \sqrt{2 n}$ слабо сходится к распределению случайной величины $\xi$, имеющей гамма-распределение с параметром 2 , плотностью

$$
p_{\xi}(x)=x e^{-x}, \quad x>0,
$$

и характеристической функцией

$$
\varphi_{\xi}(t)=(1-i t)^{-2} .
$$

Таким образом, достаточно убедиться в том, что при $n \rightarrow \infty$ для любого фиксированного $t \in \mathbf{R}$

$$
\varphi_{\xi_{n} / \sqrt{2 n}}(t)=\mathbf{E}\left(\exp \left(i t \xi_{n} / \sqrt{2 n}\right)\right)=(1-i t)^{-2}(1+o(1)) .
$$

Положим $|u|=1$ и $|z|=z_{n} \in(0,1)$, где $z_{n}-$ корень уравнения (49), тогда $0<|u z|=z_{n}<1$, и поэтому согласно (13), (61)

$$
\begin{aligned}
\mathbf{E} u^{\xi_{n}} & =\sum_{k=1}^{n} \mathbf{P}\left\{\xi_{n}=k\right\} u^{k} \\
= & \frac{1}{L(n)}\left(L(n-1) u+\frac{(n-1) !}{4 \pi i} \oint_{|z|=z_{n}} \frac{\exp \left((z / 2)\left(1+(1-z)^{-1}\right)\right)}{z^{n+1}}\left(\sum_{k=2}^{\infty} k(u z)^{k}\right) d z\right) \\
= & \frac{1}{L(n)}\left(L(n-1) u+\frac{(n-1) !}{4 \pi i} \oint_{|z|=z_{n}} \frac{\exp \left((z / 2)\left(1+(1-z)^{-1}\right)\right)}{z^{n+1}} \frac{u^{2} z^{2}(2-u z)}{(1-u z)^{2}} d z\right) .
\end{aligned}
$$


Как показано в доказательстве предложения 5 , при $n \rightarrow \infty$

$$
z_{n}=1-\frac{1}{\sqrt{2 n}}+O\left(n^{-1}\right)
$$

и если при $t \in \mathbf{R}$ положить

$$
u=\exp \left(\frac{i t}{\sqrt{2 n}}\right)=1+\frac{i t}{\sqrt{2 n}}+O\left(\left(n^{-1}\right)\right),
$$

To

$$
\begin{aligned}
|u| & =1, \\
\left(1-u z_{n}\right)^{-2} & =2 n(1-i t)^{-2}(1+O(1 / \sqrt{n})), \\
u^{2} z_{n}^{2}\left(2-u z_{n}\right) & =1+O(1 / \sqrt{n}) .
\end{aligned}
$$

Используя (48) и подставляя полученные оценки в (63), получаем (62).

Теорема 12 доказана.

Замечание 6. Тем же методом нетрудно доказать, что при $n \rightarrow \infty$

$$
\begin{aligned}
& \mathbf{E} \xi_{n}=\sqrt{8 n}(1+o(1)), \\
& \mathbf{D} \xi_{n}=4 n(1+o(1)) .
\end{aligned}
$$

\section{Список литературы}

1. Сачков В. Н., Введение в комбипаториые методы дискретной математики. Наука, Москва, 1982.

2. Holst L., On numbers related to partition of unlike objects and occupancy problems. European $J$. Comb. $19812,231-237$.

3. Колчин В. Ф., Севастьянов Б. А., Чистяков В. П., Случайные размещения. Наука, Москва, 1976.

4. Бритиков В. Е., Асимптотика числа лесов из некорневых деревьев. Матем. заметки (1988) 43, №5, 672-684.

5. Бритиков В. Е., Предельное поведение числа деревьев заданного объема в случайном лесе из некорневых деревьев. В сб.: Вероятиостиые задачи дискретной математики. МИЭМ, Москва, 1988 , c. $7-12$.

6. Колчин В. Ф., Случайные отображения. Наука, Москва, 1984.

7. Федорюк М. В., Метод перевала. Наука, Москва, 1977.

8. Тимашев А. Н., Случайные леса с известным числом свободных деревьев. Обозрение прикладной и промышлениой матем. (2001) 8, №2, 804-806.

Статья поступила 10.07.2003. Переработанный вариант поступил 24.09.2004. 